\title{
A realidade da prática do enfermeiro em um Serviço de Diálise: confrontando aspectos das Diretrizes Curriculares Nacionais
}

\author{
The reality of nurses' practice in a Dialysis Service: confronting aspects of the National \\ Curriculum Guidelines
}

La realidad de la práctica de enfermería en un servicio de Diálisis: hacer frente a los aspectos de las Directrices Curriculares Nacionales

Luciana Mara Rosa Milagres ${ }^{1 *}$, Carolina da Silva Caram¹, Maria José Menezes Brito ${ }^{1}$, Samantha Vieira Alves Amaral ${ }^{1}$, Pâmela Malheiro Oliveira ${ }^{1}$, Camila Amurim de Souza ${ }^{1}$, Samira Alves Barbosa Gonçalves ${ }^{1}$, Mariana Rodrigues Ramos ${ }^{1}$.

\section{RESUMO}

Objetivo: Analisar a prática do enfermeiro em um serviço de diálise de um Hospital, considerando as Diretrizes Curriculares Nacionais (DCN) do curso de enfermagem. Métodos: Trata-se de uma pesquisa qualitativa realizada em um serviço de diálise de um Hospital em Minas Gerais, cujos participantes foram 9 enfermeiros. A coleta de dados foi realizada mediante observação e análise documental das DCN do curso de Enfermagem. A pesquisa foi aprovada pelo Comitê de Ética em Pesquisa. Resultados: Percebeu-se que o enfermeiro assume papel de liderança, mas, as atividades burocráticas são realizadas em detrimento da assistência; as tecnologias em saúde precisam ser articuladas e; o relacionamento interpessoal e o trabalho em equipe multiprofissional precisa ser valorizado. Conclusão: A realidade demonstra que os enfermeiros nem sempre agem em consonância com as DCN, sendo importante rever as atribuições do enfermeiro, visando favorecer a qualidade do cuidado ao paciente e estar em consonância com o preconizado na formação desses profissionais.

Palavras-Chave: Enfermagem, Nefrologia, Educação em Enfermagem, Conhecimentos, Atitudes e Prática em Saúde.

\section{ABSTRACT}

Objective: to analyze nurses' practice in a dialysis service of a Hospital, considering the National Curricular Guidelines (NCD) of the nursing course. Method: This is a qualitative study performed in a dialysis service of a Hospital in Minas Gerais, whose participants were 9 nurses. The data collection was carried out observation and NCD documentary analysis of the nursing course. The research was approved by the Ethics Research Committee. Results: It was noticed that nurses assumes a leadership role, but bureaucratic activities are often performe to the detriment of care; Health care technologies need to be articulated, and the interpersonal relationship and the multiprofessional team work needs to be valued. Conclusion: The reality shows that nurses do not always act in agreement with NCD, and it is important to review nurses' assignments, in order to favor quality of patient care and to be in line with what is recommended in the training of these professionals.

Key words: Nursing, Nephrology, Nursing Education, Knowledge, Attitudes, Practice off health.

${ }^{1}$ Universidade Federal de Minas Gerais (UFMG). Belo Horizonte MG. *E-mail: lucianarosamilagres@gmail.com

SUBMETIDO EM: 5/2019

ACEITO EM: 6/2019

PUBLICADO EM: 7/2019

REAS/EJCH | Vol. 11(11) | e907 | DOI: https://doi.org/10.25248/reas.e907.2019 Página 1 de 8 


\section{RESUMEN}

Objetivo: analizar la práctica de enfermería en el servicio de diálisis del Hospital, teniendo en cuenta las Directrices Curriculares Nacionales del curso de enfermería. Método: Estudio cualitativo realizado en un servicio de diálisis del Hospital de Minas Gerais, cuyos participantes 9 enfermeras. La recolección de datos se realizó mediante la observación y el análisis documental de DCN, por supuesto, de enfermería. La investigación fue aprobada por el Comité de Ética de la Investigación. Resultados: Se observó que asume el papel de liderazgo de enfermería, pero son actividades que se realizan a menudo de la ONU, a expensas de la atención; Tecnologías de la salud deben ser articulados, y la relación interpersonal y trabajo en equipo multiprofesional debe ser valorado. Conclusión: La realidad muestra Que enfermeras no siempre actúan en armonía con DCN, y es importante para revisar las asignaciones de las enfermeras, con el fin de complacer a la calidad de la atención al paciente y para estar en línea con lo que se recomienda en la educación de enfermería.

Palabras clave: Enfermería, Nefrología, Educación en Enfermería, Conocimientos, Actitudes y Práctica en Salud.

\section{INTRODUÇÃO}

O aumento da abrangência da educação superior vem se dando, fundamentalmente, pelo crescimento da oferta de instituições de ensino e, consequentemente, de cursos e vagas oferecidas. Essa expansão apresenta relação direta com o desenvolvimento econômico e social do país, uma vez que o Brasil apresenta baixa proporção de estudantes, na faixa etária relevante, matriculados no ensino superior (TEIXEIRA E et al., 2013).

Dessa forma a área da formação do enfermeiro acompanha essa temática de aumento, porém é necessário destacar que a formação em enfermagem não garante ao profissional o senso crítico e reflexivo no atendimento integral da população conforme preconizado nas Diretrizes Curriculares Nacionais do Curso de Graduação em Enfermagem. E a cada dia o mercado de trabalho exige um contingente de profissionais para atender as necessidades de saúde da população (TEIXEIRA E et al., 2013).

Assim a atuação do enfermeiro deve articular diferentes habilidades e competências visando o trabalho em equipe e a garantia da qualidade em prol da eficiência e eficácia da assistência prestada. E é fundamental que o enfermeiro contemporâneo enfrente os desafios e esteja preparado para a diversidade e complexidade do mundo atual. Nesse sentido, a prática do enfermeiro envolve múltiplas ações, como o gerenciar, o cuidar e o educar (OLIVEIRA NB, COSTA E SILVA FV, ASSAD LG; 2015; FERNADES JD; REBOUÇAS LC, 2013).

$\mathrm{Na}$ gama de especialidades que são ofertadas para o profissional enfermeiro a nefrologia se destaca visto que o desenvolvimento dessa como especialidade está atrelado ao aprimoramento das técnicas de terapia renal substutiva como hemodiálise e dialise peritoneal. Nesse sentido, os enfermeiros necessitaram aprender o manejo dos equipamentos e a melhor maneira de cuidar da clientela com insuficiência renal crônica (OLIVEIRA NB, COSTA E SILVA FV, ASSAD LG; 2015).

O fazer do enfermeiro especialista em nefrologia é representado pelo cuidado paliativo, o qual é mediado por tecnologias tangíveis através de equipamentos de última geração, sendo desenvolvido dentro de unidades de diálise/hemodiálise com pacientes no estágio final da insuficiência renal crônica. A assistência prestada pelo enfermeiro nesse setor é ampla e envolve a atuação em equipe multiprofissional, além do gerenciamento do cuidado a ser prestado pela equipe de enfermagem. Para tanto, o enfermeiro deve adotar competências e habilidades gerais e específicas estabelecidas pelas Diretrizes Curriculares Nacionais (DCN) (OLIVEIRA NB, COSTA E SILVA FV, ASSAD LG; 2015; VIEIRA MA et al., 2016).

A Lei de Diretrizes e Bases da Educação (LDB), Lei no 9.394 de 20 de dezembro de 1996, instituiu as DCN como forma de traçar a diretiva das bases educacionais da Educação Profissional, baseada no desenvolvimento de competências, habilidades e conhecimentos que os profissionais precisam desenvolver 
na graduação para atuarem no mercado de trabalho (BRASIL, 1996). Por competências profissionais entende-se a capacidade de articular, mobilizar, colocar em ação valores, habilidades e conhecimentos necessários para o desempenho eficaz de práticas requeridas pela natureza do trabalho (BRASIL, 1999).

$\mathrm{Na}$ graduação em enfermagem, as DCN apontam para o exercício das habilidades e competências essenciais dos profissionais de saúde, sendo elas: liderança, tomada de decisões, atenção à saúde, comunicação efetiva, gerenciamento das atividades de educação permanente (BRASIL, 2001). Além das competências e habilidades gerais citadas acima, as DCN determinam, também, as competências e habilidades específicas que devem ser desenvolvidas pelo enfermeiro para sua atuação profissional, sendo essenciais para a prática no âmbito dos serviços de saúde e, também, no serviço de diálise (OLIVEIRA NB, COSTA E SILVA FV, ASSAD LG; 2015).

Nesse ínterim, vale ressaltar que a atuação do enfermeiro na nefrologia é fundamental para o sucesso do tratamento/cuidado prestado ao paciente, bem como para a redução da exposição do paciente a riscos. Dessa forma, é relevante conhecer quais competências e habilidades específicas são mobilizadas pelos enfermeiros na prática em diálise, no sentido de aprimorá-las, traduzindo em benefícios para os pacientes e para o serviço. Além disso, pode suscitar questões acerca da formação acadêmica dos enfermeiros que atuam nos serviços de nefrologia, a fim de possibilitar o desenvolvimento técnico e clínico desses profissionais e possibilitar a reflexão da prática cotidiana. Visto o exposto, o objetivo deste estudo foi analisar a prática do enfermeiro em um serviço de diálise de um Hospital, considerando as Diretrizes Curriculares Nacionais do curso de graduação em enfermagem.

\section{METODOLOGIA}

Trata-se de um estudo com abordagem qualitativa desenvolvido em um serviço de diálise privado, localizado em um Hospital na cidade de Belo Horizonte, Minas Gerais.

Os participantes desse estudo foram 09 enfermeiros que atuam no serviço de diálise, cenário deste estudo. Estabeleceu-se como critério de inclusão os enfermeiros que atuam na assistência direta ao paciente no serviço de diálise, que estavam presentes no momento da coleta de dados, excluindo então, os que atuavam apenas na coordenação da equipe e aqueles que não aceitaram participar da pesquisa. Os participantes foram esclarecidos dos objetivos do estudo e, para o acompanhamento das atividades em todas as circunstâncias foi solicitada a assinatura do Termo de Consentimento Livre e Esclarecido (TCLE).

A coleta de dados foi realizada por meio da observação, enfocando o cenário e as práticas desenvolvidas por enfermeiros no cotidiano de trabalho e análise documental das DCN do curso de enfermagem. O período da coleta foi de abril a maio de 2018.

Assim, a realização da observação foi organizada em três etapas essenciais, através de um roteiro sistematizado. A primeira fase consistiu na aproximação do pesquisador com o grupo de enfermeiros da diálise. A segunda fase foi a observação propriamente dita, em que o pesquisador captou a visão do conjunto dos participantes, suas ações, relações e práticas no cotidiano de trabalho. As observações foram anotadas em diários de campo em forma de notas de campo organizadas por local, data e horário, constando o tipo de atividade observada, a descrição do fato e as impressões do observador. A terceira fase ocorreu após a coleta dos dados, em que eles foram sistematizados e organizados.

Após a coleta de dados, foi feita a "ordenação dos dados" das observações anotadas em diários de campo. As observações foram submetidas a uma releitura em que as informações correspondentes às competências e habilidades específicas para atuação do enfermeiro foram mapeadas e sublinhadas. As observações foram codificadas em relação à competência e habilidade disposta pela $\mathrm{DCN}$ mobilizada ou não para cada atividade observada.

A pesquisa foi analisada e aprovada pelo Comitê de Ética em Pesquisa de Seres Humanos da Universidade Federal de Minas Gerais - Parecer ํㅡ 682.671 em conformidade com a Resolução do Conselho Nacional de Saúde 466/12. 


\section{RESULTADOS E DISCUSSÃO}

Fizeram parte do estudo 9 (100\%) enfermeiros colaboradores do serviço de nefrologia do hospital cenário do estudo, destes 5 (56 \%) eram do sexo feminino, a faixa etária entre 20 a 29 anos foi a maior parte 4 (44\%). Quanto ao tempo de formação a grande parte com 6 a 17 meses 4(44\%), todos com especialização lato sensu em nefrologia, e a maioria 5 (56 \%) tinha entre 6 a 23 meses de tempo de trabalho na intuição (Tabela 1).

Tabela 1 - Distribuição dos participantes quanto ao sexo, faixa etária, tempo de formação e tempo de trabalho na instituição. Belo Horizonte, 2019.

\begin{tabular}{|c|c|c|}
\hline Variável & $\mathbf{N}$ & $\%$ \\
\hline \multicolumn{3}{|l|}{ Sexo } \\
\hline Feminino & 5 & $56 \%$ \\
\hline Masculino & 4 & $44 \%$ \\
\hline Total & 9 & $100 \%$ \\
\hline \multicolumn{3}{|l|}{ Faixa etária } \\
\hline $20 \vdash 30$ anos & 4 & $44 \%$ \\
\hline $30+40$ anos & 3 & $34 \%$ \\
\hline$\geq 40$ anos & 2 & $22 \%$ \\
\hline Total & 9 & $100 \%$ \\
\hline \multicolumn{3}{|c|}{ Tempo de formação } \\
\hline $6 \vdash 18$ meses & 4 & $44 \%$ \\
\hline 18 - 30 meses & 3 & $34 \%$ \\
\hline$\geq 30$ meses & 2 & $22 \%$ \\
\hline Total & 9 & $100 \%$ \\
\hline \multicolumn{3}{|c|}{ Tempo de trabalho na instituição } \\
\hline $6 \vdash 24$ meses & 5 & $56 \%$ \\
\hline $24-42$ meses & 2 & $22 \%$ \\
\hline$\geq 42$ meses & 2 & $22 \%$ \\
\hline Total & 9 & $100 \%$ \\
\hline
\end{tabular}

Fonte: Dados da pesquisa, 2019.

Tendo por base os dados coletados e a ordenação das observações em torno do eixo temático surgiram cinco categorias com os seguintes títulos: "Atividades burocráticas e cuidado holístico e humanizado", "Competências gerenciais", "Interação com a equipe multiprofissional e éticas nas relações profissionais", " Relacionamento interpessoal', "Qualificação profissional e produção do conhecimento no serviço de diálise".

\section{Atividades burocráticas e cuidado holístico e humanizado}

Observou-se que o enfermeiro prioriza o trabalho burocrático e de coordenação em sua prática cotidiana no serviço de nefrologia em detrimento da assistência direta ao paciente. O enfermeiro, no contexto do cenário deste estudo, pouco se encontra com o paciente, não priorizando, portanto, o cuidar. Foi percebido, em diversos momentos, que os enfermeiros relutaram quando foram solicitados a realizar procedimentos e intervenções junto aos pacientes sob sua responsabilidade, privilegiando o trabalho burocrático e de supervisão.

Ainda nessa temática não se percebe na atuação dos enfermeiros a preocupação em oferecer assistência integral e holística ao paciente.

A atuação é voltada para os sinais e sintomas, considerando a insuficiência renal e o processo dialítico em si, não havendo planos de cuidado relacionados a outros agravos, patologias decorrentes ou não da terapia dialítica e na valorização da pessoa humana.

Diante disso, essa situação entra em conflito com os Incisos I, II, VI, VII e XI, da DCN (BRASIL, 2001) são eles: 
I - Atuar profissionalmente, compreendendo a natureza humana em suas dimensões, em suas expressões e fases evolutivas;

II - Incorporar a ciência/arte do cuidar como instrumento de interpretação profissional;

VI - Reconhecer a saúde como direito e condições dignas de vida e atuar de forma a garantir a integralidade da assistência, entendida como conjunto articulado e contínuo das ações e serviços preventivos e curativos, individuais e coletivos, exigidos para cada caso em todos os níveis de complexidade do sistema;

VII - Atuar nos programas de assistência integral à saúde da criança, do adolescente, da mulher, do adulto e do idoso;

$X I$ - Responder às especificidades regionais de saúde através de intervenções planejadas estrategicamente, em níveis de promoção, prevenção e reabilitação à saúde, dando atenção integral à saúde dos indivíduos, das famílias e das comunidades.

Os enfermeiros se centram mais no desenvolvimento das atividades administrativas e burocráticas relacionadas à organização e estruturação do serviço de enfermagem, do que no cuidado ao paciente. Embora seja de conhecimento que $o$ ato de cuidar é influenciado por questões organizacionais e do processo de trabalho, é importante que o enfermeiro desenvolva sua prática em consonância com os princípios da sua profissão e junto ao paciente. Nesse sentido, o cuidar é uma atitude, isto é, o cuidar demonstra uma atitude de ocupação, preocupação e responsabilização com o paciente, sendo necessário que as atividades burocráticas não sobreponham o cuidado direto, mas que se complementem em prol da assistência segura e holística (SALVIANO MEM, et al., 2016; MORORÓ DDS, et al., 2017).

Logo a ação do cuidar vai ao encontro do compromisso de manter a dignidade e a singularidade do ser cuidado. É um momento de preocupação, interesse e motivação, em que o respeito, a consideração e a gentileza se tornam diferenciais. A consciência do cuidado deve abranger a capacidade de decisão, a sensibilidade e o pensamento crítico, para diferenciar o cuidado da realização de procedimentos, é fundamental que o enfermeiro desenvolva as competências gerenciais em consonância com a prática do cuidado ao paciente (SALVIANO MEM, et al., 2016; MORORÓ DDS, et al., 2017).

\section{Competências gerenciais}

Observou-se que o enfermeiro é reconhecido como coordenador da equipe, tanto pelos técnicos de enfermagem como pelos pacientes/familiares, sendo considerado referência no setor. $O$ enfermeiro adequa os cuidados prestados pela equipe de técnicos de enfermagem às necessidades apresentadas pelos pacientes e demandas médicas. Além disso, organiza as diálises que são realizadas em outros setores do hospital, bem como organiza a escala dos funcionários a fim de prestar os serviços demandados.

Em face da observação, a prática do enfermeiro é condizente com o que é preconizado pelos Incisos XII, XIX, XX, XXVIII da DCN (BRASIL, 2001).

$$
\begin{aligned}
& \text { XII - Reconhecer-se como coordenador do trabalho da equipe de enfermagem; } \\
& \text { XIX - Coordenar o processo de cuidar em enfermagem, considerando contextos e } \\
& \text { demandas de saúde; } \\
& \text { XX-Prestar cuidados de enfermagem compatíveis com as diferentes necessidades } \\
& \text { apresentadas pelo indivíduo, pela família e pelos diferentes grupos da comunidade; } \\
& \text { XXVIII - Interferir na dinâmica de trabalho institucional, reconhecendo-se como } \\
& \text { agente desse processo. }
\end{aligned}
$$

O enfermeiro é considerado, nos serviços de saúde, como líder por desenvolver diversas atividades, tanto para a organização do setor quanto para a equipe de saúde. 
A função gerencial é importante para desenvolver as competências do enfermeiro como liderança, trabalho em equipe, comunicação, relacionamento interpessoal, tomada de decisão, planejamento e organização, dentre outras competências necessárias ao seu perfil profissional (MORORÓ DDS, et al., 2017)

Apesar de o enfermeiro ser a liderança no setor de diálise, percebeu-se que a liderança encontra atrelada a questões burocráticas do serviço, organizando o cuidado de acordo com a demanda e o processo de trabalho de forma geral. Contudo, esse cuidado é considerado indireto, visto que eles priorizaram as tarefas administrativas em detrimento da assistência, como visto anteriormente. Dessa forma, realizar o cuidado significa que o enfermeiro precisa ter habilidade de articular o trabalho em equipe e as demandas do paciente, visando o sujeito cuidado, isto é, atuando como líder (GELBCKE FL, et al., 2009).

\section{Interação com a equipe multiprofissional e éticas nas relações profissionais}

Conforme as observações realizadas a equipe que atende no cenário do estudo incluem nutricionista, assistente social, farmacêutico, psicólogo, médico, enfermeiro e técnico de enfermagem. Entretanto, o trabalho multiprofissional não é efetivado, haja vista que as ações são fragmentadas entre as categorias profissionais.

Nota-se através dos aspectos avaliados que os instrumentos para a coleta de dados dos pacientes não são integrados, o que leva a repetição de informações, tornando o processo dispendioso tanto para o profissional, que precisa levantar dados relativos à terapia que estão separados fisicamente por prontuários diferenciados, quanto para o paciente que verbaliza, várias vezes, as mesmas queixas e situações vivenciadas para os profissionais. Assim, muitas informações são perdidas ou não utilizadas de forma eficiente e, consequentemente, o trabalho não é integrado.

Além disso os profissionais apresentaram condutas éticas inapropriadas como: questionavam condutas de colegas de trabalho no salão de diálise durante os turnos de tratamento, bem como queixavam sobre o processo de trabalho na presença de pacientes. Presenciou-se diálogos entre enfermeiros sobre condutas e posturas dos técnicos de enfermagem, pacientes e assuntos pessoais no salão durante as sessões de diálise.

Diante disso, percebe-se que ainda precisa de esforços por parte da equipe para a efetivação do trabalho multiprofissional, conforme disposto pelas DCN (BRASIL, 2001).

\section{XXII - Integrar as ações de enfermagem às ações multiprofissionais;}

VIII- Ser capaz de diagnosticar e solucionar problemas de saúde, de comunicar-se, de tomar decisões, de intervir no processo de trabalho, de trabalhar em equipe e de enfrentar situações em constante mudança;

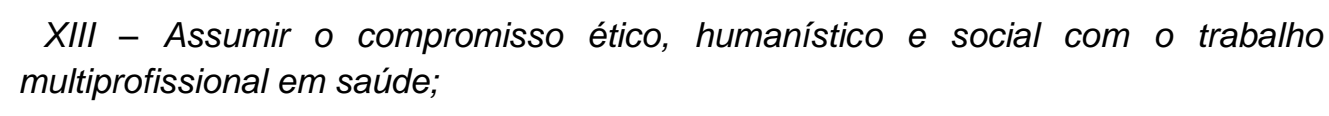

XIII - Assumir o compromisso ético, humanístico e social com o trabalho multiprofissional em saúde;

XXVII - Respeitar os princípios éticos, legais e humanísticos da profissão.

No trabalho em saúde é indispensável atuar de modo complementar e multiprofissional. No caso deste estudo, percebe-se a fragmentação da atuação dessa equipe, retardando e dificultando o processo de trabalho. Assim, atuar sem interação faz com que as individualidades se percam e sejam negligenciadas, relativizando o cuidado. O cuidado de excelência é o objetivo principal de uma organização de saúde, sendo importante que os profissionais atuem em equipe com ética profissional para alcançar os objetivos do serviço, atendendo às demandas dos pacientes e baseados no bom relacionamento interpessoal (NASCIMENTO KC, et al., 2008).

Nesse ínterim, vale ressaltar que dentre as diretrizes elucidadas pela Política Nacional de Humanização (PNH) a Clínica Ampliada representa uma maneira de enfrentamento, pois considera que a complexidade do trabalho em saúde necessita da abordagem de uma equipe multiprofissional. Com isso, espaços onde a fragmentação do processo de trabalho possa ser discutida possibilitam aos trabalhadores refletirem acerca da compreensão ampliada do processo saúde-doença, do compartilhamento de diagnósticos e intervenções e do trabalho em saúde organizado na lógica das equipes de referência e de apoio matricial. 
O trabalho em equipe multiprofissional representa, dessa forma, uma maneira de os profissionais atingirem a prática do cuidado humanizado (SANTOS JLG, et al., 2013).

\section{Relacionamento interpessoal}

Os enfermeiros demonstraram ter relação afetiva entre eles, compartilhando vivências e estreitando os laços. Nos horários de convivência como, por exemplo, na troca de plantão e no intervalo do lanche, tanto entre os enfermeiros quanto entre eles e os técnicos de enfermagem surgem assuntos casuais, demonstrando que as relações ultrapassam as barreiras profissionais.

Essa relação não acontece somente entre os profissionais, mas também, entre eles e pacientes/familiares. Observou-se que os profissionais estabelecem vínculo com os familiares, o qual demostrou favorecer a confiança do paciente/familiares com o profissional, fazendo com que eles aceitem melhor as intervenções e as mudanças de terapia, criando um ambiente agradável e facilitando a atuação na assistência.

Entretanto, em alguns momentos, o vínculo prejudicou o fazer profissional, pois a intimidade extrapolou a relação paciente-enfermeiro, permitindo que o paciente fizesse exigências não cabíveis com a realidade do serviço, como por exemplo, pedir para parar a terapia para montar computador, comer escondido durante a sessão, entrar com acompanhante, reduzir o tempo de diálise, entre outros. Visto o exposto, é possível relacionar as observações com os Incisos III e IX das DCN (BRASIL, 2001):

\section{IX - Reconhecer as relações de trabalho e sua influência na saúde; \\ III - Estabelecer novas relações com o contexto social, reconhecendo a estrutura e as formas de organização social, suas transformações e expressões.}

O paciente que está sendo cuidado não é um ser isolado e sim compreendido como uma extensão social e familiar e quando em situação de vulnerabilidade o equilíbrio e os papeis de cada membro se alteram, sendo fundamental atender as necessidades de toda a rede familiar. Logo o vínculo enfermeiro-paciente-família é essencial. Um bom relacionamento permite uma confiança que facilita as práticas de educação em saúde, para tanto o enfermeiro deve ter o discernimento e uma adequada forma de comunicação (FRAZÃO, CMFQ et al., 2014).

\section{Qualificação profissional e produção do conhecimento no serviço de diálise}

Os enfermeiros que atuam nesse serviço de diálise se especializaram em nefrologia. Eles são responsáveis pelo acompanhamento de indicadores de enfermagem referentes à qualidade da assistência e, caso necessário, criam e desenvolvem planos de ação para adequar a realidade aos índices preconizados, buscando melhorar a assistência prestada pelo serviço, aplicando o conhecimento especializado na prática. Apesar dessa iniciativa, no período de realização do estudo, nenhum projeto de pesquisa científica estava sendo realizado no setor, demonstrando pouca interação entre a academia científica com a prática do cotidiano. Na enfermagem, a pesquisa é parte importante do processo de revisão, proposição e testagem de conceitos, significados, teorias, modelos e processos de cuidar reafirmando a arte e reconstruindo a ciência do cuidado (FERREIRA MA, et al., 2014).

Não se observou a atuação deles em projetos de pesquisa, apesar da unidade de diálise gerar dados passíveis de verificação e, consequentemente, produção de conhecimento. Porém os enfermeiros buscam a qualificação profissional e a utilização do conhecimento na prática, o que é condizente com os incisos IV e XXVI (BRASIL, 2001).

\section{IV - Desenvolver formação técnico científica que confira qualidade ao exercício profissional;}

XXVI-Desenvolver, participar e aplicar pesquisas e/ou outras formas de produção de conhecimento que objetivem a qualificação da prática profissional;

A pesquisa cientifica é uma importante metodologia para concretização da enfermagem como ciência e a busca contínua de práticas baseadas em evidências para o enfermeiro nefrologista (OLIVEIRA NB et al 2015). 


\section{CONSIDERAÇÕES FINAIS}

As DCN norteiam a organização dos currículos das Instituições de Educação superior no Brasil na busca de um perfil profissional adequado. No entanto a realidade demonstra que os enfermeiros nem sempre agem em consonância com as DCN, sendo importante rever suas atribuições, visando favorecer a qualidade do cuidado ao paciente e estar em consonância com o preconizado na formação desses profissionais. Dessa forma, essa pesquisa levanta a necessidade de revisão das atribuições do enfermeiro enquanto organizador e prestador da assistência.

\section{REFERÊNCIAS}

1. BRASIL. Ministério da Educação. Conselho Nacional de Educação. Câmara de Educação Superior. Resolução CNE/CES N. 3, de 07 de novembro de 2001. Institui as Diretrizes Curriculares Nacionais do Curso de Graduação em Enfermagem. Diário Oficial da República Federativa da União. Brasília, 09 nov. 2001. Seção 1, p. 37. Brasília (DF): Ministério da Educação e Cultura; 2001.

2. BRASIL. Ministério da Saúde. Conselho Nacional de Saúde. Resolução n466 /12. Diretrizes e normas regulamentadoras de pesquisas envolvendo seres humanos. Diário Oficial da República Federativa do Brasil. Brasília, 2012.

3. BRASIL. Ministério da Educação. Lei no 9.394, de 20 de dezembro 1996. Estabelece as diretrizes e bases da educação nacional. Brasília, dezembro de 1996. Diário Oficial da República Federativa do Brasil, 23 Dez 1996.

4. BRASIL. Ministério da Educação. Conselho Nacional de Educação. Resolução no 4 de 22 Dez 1999: Diretrizes curriculares nacionais para a educação profissional de nível técnico. Diário Oficial da República Federativa do Brasil, 22 Dez 1999.

5. FERNANDES JD, REBOUÇAS LC. Uma década de Diretrizes Curriculares Nacionais para a Graduação em Enfermagem: avanços e desafios. Rev Bras Enferm, 2013;66(esp):95-101.

6. FERREIRA MA. Enfermagem: arte e ciência do cuidado. Esc. Anna Nery Rev. Enferm, 2011;15(4):664-666.

7. Frazão CMFQ, et al. Cuidados de enfermagem ao paciente renal crônico em hemodiálise. Rev Rene. 2014; 15(4):7019.

8. GELBCKE FL, et al. Liderança em ambientes de cuidados críticos: reflexões e desafios à Enfermagem Brasileira. Rev. bras. enferm. 2009;62 (1):1369.

9. MORORÓ DDS, et al. Análise conceitual da gestão do cuidado em enfermagem no âmbito hospitalar. Acta Paul Enferm. 2017; 30(3):323-32.

10. NASCIMENTO KC, et al. Sistematização da assistência de enfermagem: vislumbrando um cuidado interativo, complementar e multiprofissional. Rev. Esc. Enferm. USP. 2008; 42(4):643-648.

11. OLIVEIRA NB, COSTA E SILVA FV, ASSAD LG. Competências do enfermeiro especialista em nefrologia. Rev enferm UERJ, 2015; 23(3): 375-80.

12. QUEIROZ DT, et al. Observação participante na pesquisa qualitativa: conceitos e aplicações na área da saúde. Rev. enferm. UERJ. 2007; 15(2):276 - 83.

13. SALVIANO MEM, et al. Epistemologia do cuidado de enfermagem: uma reflexão sobre suas bases. Rev Bras Enferm. $201 ; 69(6): 1240-5$.

14. SANTOS JLG, et al. Práticas de enfermeiros na gerência do cuidado em enfermagem e saúde: revisão integrativa. Rev. bras. Enferm, 2013;66(2): 257263.

15. TEIXEIRA E et al. Panorama dos cursos de Graduação em Enfermagem no Brasil na década das Diretrizes Curriculares Nacionais. Rev Bras Enferm, 2013;66(esp):102-10.

16. VIEIRA MA, et al. Diretrizes Curriculares Nacionais para a área da enfermagem: o papel das competências na formação do enfermeiro. Revista Norte Mineira de Enfermagem. 2016; 5(1): 105-121. 